\title{
The effect of resist on the transfer of line-edge roughness spatial metrics from mask to wafer
}

\author{
Patrick P. Naulleau ${ }^{1}$ and Gregg M. Gallatin ${ }^{2}$ \\ ${ }^{1}$ Center for X-Ray Optics, Lawrence Berkeley National Laboratory, Berkeley, CA 94720 \\ ${ }^{2}$ Applied Math Solutions, Newtown, CT 06470
}

\begin{abstract}
Mask contributors to line-edge roughness (LER) have recently been shown to be an issue of concern for both the accuracy of current resist evaluation tests as well the ultimate LER requirements for the 22-nm production node. More recently, it has been shown that the power spectral density of the maskinduced roughness, is markedly different than that of intrinsic resist roughness and thus potentially serves as a mechanism for distinguishing mask effects from resist effects in experimental results. Further considering stochastic resist effects, however, demonstrates that such a test would only be viable in cases where the resist effects are completely negligible in terms of their contribution to the total LER compared to the mask effects. The results presented here lead us to the surprising conclusion that it is indeed possible for mask contributors to be the dominant source of LER while the spatial characteristics of the LER remain indistinguishable from the fractal characteristics of resistinduced LER.
\end{abstract}

Keyword: extreme ultraviolet, lithography, multilayer, mask, line-edge roughness

This work was supported by the Director, Office of Science, Office of Basic Energy Sciences, of the U.S. Department of Energy under Contract No. DE-AC02-05CH11231. 


\section{Introduction}

Mask errors such as pattern line-edge roughness (LER) [1] and reflective multilayer roughness [2, 3] have recently been shown [4] to potentially limit the accuracy of resist LER measurements in advanced EUV development tools. It has also been shown [5] that the mask errors affect the shape of the power spectral density (PSD) and therefore LER spatial metrics such as correlation length and roughness exponent [6-8]. The significant change in morphology of the PSD has been suggested [5] to provide a mechanism to distinguish between mask-induced LER and intrinsic resist LER. The feasibility and resolution of the PSD-based root-cause analysis approach, however, further depends on how the resist itself transfers the aerial image roughness studied in Ref. [5].

Here we explicitly consider the transfer of mask-induced LER from the aerial image to the deprotection image considering both LER magnitude and spatial scaling characterization functions: PSD height-height correlation function, and sigma versus length function. The transfer is considered both with and without stochastic effects. With the addition of stochastic effects, the resist LER component is effectively added to the model and we study how this further changes the measured metrics and consider the feasibility of distinguishing mask and resist effects in practice.

\section{Modeling overview}

The aerial-image modeling method used in this study has previously been described in the literature $[4,5]$ and is only briefly summarized here. Thin mask modeling is used with the multilayer surface roughness being represented as a random phase error in the clear area of the pattern. Because the study of LER requires the modeling of a large two dimensional area with high resolution both in the mask plane and the wafer plane full three-dimensional modeling is not feasible. For example, to compute the $2 \times 2 \mu \mathrm{m}$ image-plane lateral area required for accurate LER characterization and the depth structure of the EUV mask as would be required to model the 
multilayer roughness, the memory requirement for the $5 \times$ mask of interest here would be well over a terabyte. In the thin mask model, the absorber is modeled as infinitesimally thin and the multilayer is modeled as a random phase plane to represent the replicated multilayer roughness. This approach for multilayer roughness or defect modeling is known as the single-surface approximation [9] and has been shown to be quite effective. In the thin mask model, the entire mask is decomposed to a single complex-valued plane.

Figure 1 shows a three-dimensional representation of an example mask including the two roughness components of concern. Both the absorber LER and multilayer surface roughness are greatly exaggerated for illustrative purposes. The absorber LER can be thought of a lateral roughness whereas the multilayer roughness is longitudinal. The multilayer reflectivity is assumed to be uniform over the surface with the only effect of the roughness being a phase shift imparted upon reflection. The single-surface approximation treats the light field as being reflected directly from the top surface of the multilayer thus the phase shift in waves is simply determined geometrically as $2 \times$ the surface height deviation divided by the wavelength (13.5 $\mathrm{nm})$. Evidently, quite small surface perturbations can lead to significant phase shifts: a height of only $1.7 \mathrm{~nm}$ leads to a $\lambda / 4$ phase shift.

For the generation of the actual modeling mask used for the simulations to follow, the morphology of the absorber LER is set match that measured using scanning electron micrographs (SEMs) and the phase roughness morphology is determined from atomic force microscopy [4]. The flare and wavefront aberrations used in the modeling are based on published results from the SEMATECH Berkeley microfield exposure tool (MET) [10-13]. It should be noted that projection optics flare has previously been shown not to be a significant contributor to image-plane LER [4]. 


\section{Resist LER transfer without stochastics}

We begin by exploring the effect of the resist mean-field transfer function on the image plane LER, initially ignoring stochastic effects arising from the resist itself and/or photon arrival statistics. It has been demonstrated that, analogous to an optical system, the resist itself can be represented as a point-spread function (PSF) or transfer function transferring the incident aerial image to a deprotection image [14-17]. From this perspective we can see that the resist will have an effect on the mask-induced LER just as projection optics did [1-3]. The difference will simply be in the form of the PSF. The expected form of the resist deprotection PSF has been derived from the reaction diffusion equations [18] and can be represented in three dimensions as

$$
P S F_{r}(r, t)=1-\exp \left[-\frac{k t}{4 \pi R^{2}}\left(1-\operatorname{erf}\left(\frac{r}{2 R}\right)\right)\right]
$$

Where $\operatorname{erf}(\mathrm{a})$ is the error function, $t$ is the post-exposure bake time, $R$ is the diffusion range, and $k$ is the deprotection rate. To deal with two dimensional aerial images, this equation can be collapsed to the $x y$ plane by integrating along $z$ yielding an effective two-dimensional PSF.

Figure 2 shows an image of the computed aerial image intensity given a mask matching the absorber and surface statistics of a typical EUV mask [4]. The illumination condition is annular for an inner $\sigma$ of 0.35 and an outer $\sigma$ of 0.55 and the image is computed at best focus. This aerial image can then be convolved with the PSF from Eq. [1] to determine the deprotection image from which we can measure the LER and hence the effect of the resist PSF. Figures 3 and 4 show the resulting LER power spectral density (PSD) as a function of $R$ and $k$, respectively. In

Fig. 3, $k$ is assumed to be $4 \mathrm{~nm}^{3} / \mathrm{sec}$ and in Fig. $4, R$ is assumed to be $15 \mathrm{~nm}$. In both cases, $t$ is set to 90 seconds. For comparison, the plots also show the PSD of typical resist roughness which 
is self-affine in nature. Figures 5(a) and (b) show the resist PSFs as a function of radius used in to derive the PSDs in Figs. 3 and 4.

The results in Figs. 3 and 4 show that $R$ has significant effect the morphology of the LER power spectrum while the PSD is quite insensitive to $k$. It is seen that larger $R$ values dampen the abrupt drop in the PSD characteristic of mask-induced aerial-image LER. This makes it more difficult to distinguish resist-filtered mask roughness from typical resist roughness. Nevertheless differences in morphology remain. Previous analysis [5] demonstrated that one potential mechanism for distinguishing mask roughness from resist roughness was to compare the roughness exponent $(\alpha)$ as measured from the PSD and the height-height correlation function (HHCF) [7]. The method relies on the fact that resist-induced LER is expected to be self-affine in nature in which case the relationship between the rising slope of the HHCF and the falling slope of the PSD is well defined. For self-affine roughness (which, by definition, follows a power-law behavior) the roughness exponent is determined from the log-log slope of the HHCF and is related to the log-log slope of the PSD through the relationship $\alpha=\left(\right.$ Slope $\left._{\mathrm{PSD}^{-1}}\right) / 2$. Modeling has shown [5] that the mask-induced LER is not self-affine as is evident by observation of the PSD. Modeling has also shown, however, that it is difficult to distinguish mask-induced LER from self-affine roughness using the HHCF which exhibits no significant change in morphology when used to analyze mask-induced roughness instead of self-affine (resist) roughness. The slope of the PSD is much more strongly affected by the mask-induced roughness than is the slope of the HHCF, thus comparing the relationships between these two slopes (or the extracted $\alpha$ values) allows for the presence of mask-induced roughness to be observed.

Table 1 shows a comparison of the roughness exponent measured from the PSD and 
HHCF for the various resist blur parameters used in Figs. 3 and 4. Although the results show some impact of the resist blur parameters on the roughness exponent extracted from the HHCF, the effect is quite small compared to that observed from the PSD, indicating that the previously proposed method [5] for recognizing the importance mask contributors to LER remains viable in the presence of resist blur, at least in the limit of no stochastic effects from the resist (stochastic resist effects are studied in the following section). $R$ is observed to have significantly more effect on the roughness exponent than $k$. This is consistent with the PSD plots in Figs. 3 and 4. It is also interesting to note that the sensitivity of the mask-induced LER PSD to the resist blur parameters suggests the potential for an alternative method for determining these parameters based on the printing of a programmed roughness mask.

Since the resist blur has a significant impact on the PSD, one might also expect the blur to reduce the mask-induced LER magnitude. Table 1 also shows the LER values for the various blur settings. Although some effect on LER magnitude is observed, it is negligible compared to the measurement uncertainty. This is a consequence of the majority of the mask-induced LER coming from low frequencies that are not affected by the resist blur.

\section{Resist LER transfer with stochastics}

Finally we consider the effects of the resist stochastics on the total LER PSD. The resist stochastics are modeled using the methodology presented in Ref. [17]. We assume a diffusion range of $10 \mathrm{~nm}$ and deprotection rate of $4 \mathrm{~nm}^{3} / \mathrm{sec}$. We look at the resulting LER PSD as a function of resist sensitivity since the stochastics vary with sensitivity. Figure 6 shows the resulting PSDs for dose to size values of 2, 8, 32, and $128 \mathrm{~mJ} / \mathrm{cm}^{2}$. Also shown for reference is the infinite dose case which is taken from the corresponding blur case in Fig. 3. For high sensitivity cases, the characteristic morphology of mask-induced LER PSD is lost in the fractal 
resist PSD. At a dose to size somewhere between 8 and $32 \mathrm{~mJ} / \mathrm{cm}^{2}$ we begin to observe the deviation from fractal behavior indicative of mask-induced LER. We can gain even further insight into the effect of the resist stochastics on the mask-induced LER by characterizing the roughness exponent and LER as a function of sensitivity (Table 2). For dose to sizes below 16 $\mathrm{mJ} / \mathrm{cm}^{2}$, we can no longer see evidence of the mask-induced LER in the roughness exponent. The mask effects only become evident when the mask-induced LER is so dominant that it accounts for nearly $100 \%$ of the total LER. Given an uncertainty of $0.2 \mathrm{~nm}$ in the LWR, and assuming the resist-induced LWR to add in quadrature to the mask-induced LWR, we see that for the nominal 2.7-nm mask-induced LWR expected here, we would need the resist-induced LWR to be less than $1 \mathrm{~nm}$ in order to be able to detect the presence of mask-induced LWR using the previously proposed PSD method. This limit on the resist-induced LWR would further shrink if even smaller levels of mask-induced LWR were expected.

\section{Discussion}

Previous aerial-image modeling results have shown the importance of mask-induced LER and suggested methods for experimentally determining the presence of these effects. Adding stochastic resist effects to the model, however, shows the previously proposed methods only to be viable in cases where the resist effects are negligible in terms of their contribution to the total LER compared to the mask effects. Employing such a test, thus, would require the use of extremely slow resists. Moreover, the results presented here lead us to the surprising conclusion that it is indeed possible for mask contributors to be the dominant source of LER while the spatial characteristics of the LER remain indistinguishable from the fractal characteristics of resist-induced LER. 


\section{References}

1. P. Naulleau and G. Gallatin, "The line-edge roughness transfer function and its application to determining mask effects in EUV resist characterization,” Appl. Opt. 42, 3390-3397 (2003).

2. N. Beaudry, T. Milster, "Effects of mask roughness and condenser scattering in EUVL systems," Proc. SPIE. 3676, 653-662 (1999).

3. P. Naulleau, "The relevance of mask-roughness-induced printed line-edge roughness in recent and future EUV lithography tests," Appl. Opt. 43, 4025-4032 (2004).

4. P. Naulleau, D. Niakoula, G. Zhang, "System-level line-edge roughness limits in extreme ultraviolet lithography,” J. Vac. Sci. \& Technol. B 26, 1289-1293 (2008).

5. P. Naulleau and G. Gallatin, "Spatial scaling metrics of mask-induced induced line-edge roughness," J. Vac. Sci. \& Technol. B, to be published (2008).

6. D. He, F. Cerrina, "Process dependence of roughness in a positive-tone chemically amplified resist,” J. Vac. Sci. \& Technol. B 16, 3748-3751 (1998).

7. V. Constantoudis, G. P. Patsis, A. Tserepi, E. Gogolides, "Quantification of line-edge roughness of photoresists. II. Scaling and fractal analysis and the best roughness descriptors,” J. Vac. Sci. \& Technol. B 21, 1019-1026 (2003).

8. B. Bunday, M. Bishop, D. McCormack, "Determination of optimal parameters for CD-SEM measurement of line-edge roughness," Proc. SPIE 5375, 515-533 (2004).

9. E. Gullikson, C. Cerjan, D. Stearns, P. Mirkarimi, D. Sweeney, "Practical approach for modeling extreme ultraviolet lithography mask defects," J. Vac. Sci. Technol. B 20, $81-86$ (2002).

10. J. Cain, P. Naulleau, E. Gullikson, C. Spanos, "Lithographic characterization of the flare in the Berkeley 0.3-numerical aperture extreme ultraviolet microfield optic," J. Vac. Sci. \& Technol. B 24, 1234-1237 (2006). 
11. K. Goldberg, P. Naulleau, P. Denham, S. Rekawa, K. Jackson, E. Anderson and J. Liddle, “At-Wavelength Alignment and Testing of the 0.3 NA MET Optic,” J. Vac. Sci. \& Technol. B 22, 2956-2961 (2004).

12. P. Naulleau, J. Cain, K. Goldberg, "Lithographic characterization of the spherical error in an EUV optic using a programmable pupil fill illuminator,” Appl. Opt. 45, 1957-1963 (2006).

13. P. Naulleau, J. Cain, K. Goldberg, "Lithographic characterization of the field dependent astigmatism and alignment stability of a 0.3 numerical aperture EUV microfield optic," J. Vac. Sci. \& Technol. B 23, 2003-2006 (2005).

14. C. Ahn, H. Kim, K. Baik, “A novel approximate model for resist process,” Proc. SPIE 3334, 752-763 (1998).

15. J. Hoffnagle, W. Hinsberg, M. Sanchez, and F. Houle, "Method of measuring the spatial resolution of a photoresist," Opt. Lett. 27, 1776-1778, (2002).

16. P. Naulleau, "Verification of point-spread-function-based modeling of an extreme ultraviolet photoresist," Appl. Opt. 43, 788-792, (2004).

17. G. Gallatin, "Resist blur and line edge roughness," Proc. SPIE 5754, 38-52 (2005).

18. F. Houle, W. Hinsberg, M. Morrison, M. Sanchez, G. Wallraff, C. Larson, and J. Hoffnagle, "Determination of coupled acid catalysis-diffusion processes in a positive-tone chemically amplified photoresist,” J. Vac. Sci. Technol. B 18, 1874-1885 (2000). 


\section{List of Figures}

Fig. 1. Three-dimensional representation of an example mask including the two roughness components of concern. Both the absorber LER and multilayer surface roughness are greatly exaggerated for illustrative purposes.

Fig. 2. Image of computed aerial image intensity given a mask matching the absorber and surface statistics of a typical EUV mask. The illumination condition is annular for an inner $\sigma$ of 0.35 and an outer $\sigma$ of 0.55 and the image is computed at best focus.

Fig. 3. LER power spectral density measure from the aerial image after convolution with the resist PSF from Eq. [1]. Various $R$ values are considered, $k$ is assumed to be $4 \mathrm{~nm}^{3} / \mathrm{sec}$, and $t$ is 90 seconds. For comparison, the plots also show the PSD of typical resist roughness which is self-affine in nature.

Fig. 4. LER power spectral density measure from the aerial image after convolution with the resist PSF from Eq. [1]. Various $k$ values are considered, $R$ is assumed to be $15 \mathrm{~nm}$, and $t$ is 90 seconds. For comparison, the plots also show the PSD of typical resist roughness which is self-affine in nature.

Fig. 5. (a) Resist PSF as a function of $R$ as used to generate the data in Fig. 3 ( $k$ is $4 \mathrm{~nm}^{3} / \mathrm{sec}$ ). (b) Resist PSF as a function of $k$ as used to generate the data in Fig. 4 ( $R$ is $15 \mathrm{~nm}$ ). $t$ is 90 seconds in both cases.

Fig. 6. PSD after inclusion of resist stochastic effects assuming dose to size values of $2,8,32$, and $128 \mathrm{~mJ} / \mathrm{cm}^{2}$. The resist blur is set to $10 \mathrm{~nm}$ and deprotection rate is $4 \mathrm{~nm}^{3} / \mathrm{sec}$. Also shown for reference is the infinite dose case which is taken from the corresponding blur case in Fig. 3. 


\section{List of Tables}

Table 1. Comparison of the roughness exponent measured from the PSD and HHCF for the various resist blur parameters used in Figs. 3 and 4. Also shown are the corresponding LER values. Although some effect on LER magnitude is observed due to the resist blur, it is negligible compared to the measurement uncertainty.

Table 2. Roughness exponent and LER as a function of sensitivity for model including both mask and resist effects. For dose to sizes below $16 \mathrm{~mJ} / \mathrm{cm}^{2}$, we can no longer see evidence of the mask-induced LER in the roughness exponent. The mask effects only become evident when the maskinduced LER is so dominant that it accounts for nearly $100 \%$ of the total LER. 
Naulleau and Gallatin, Fig. 1

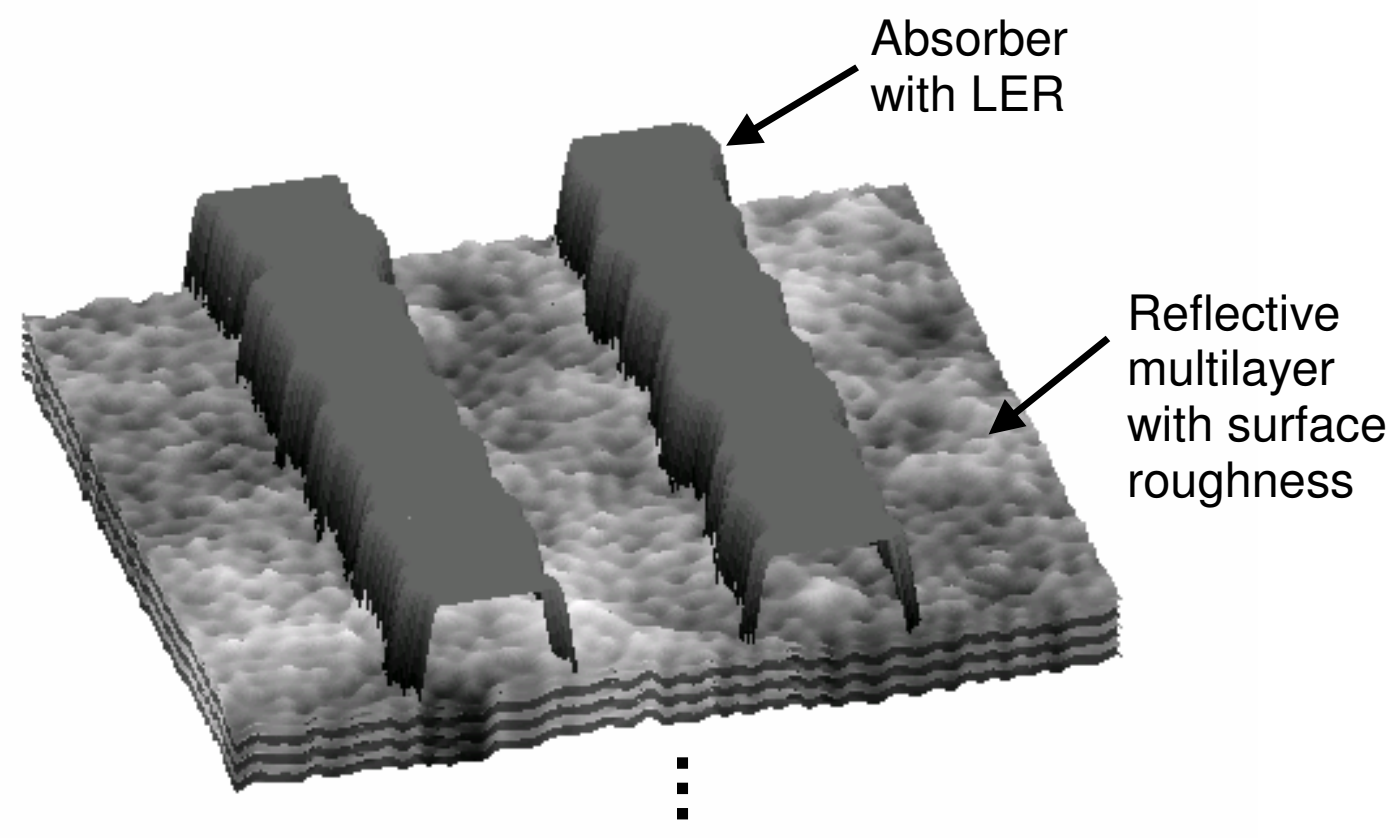


Naulleau and Gallatin, Fig. 2

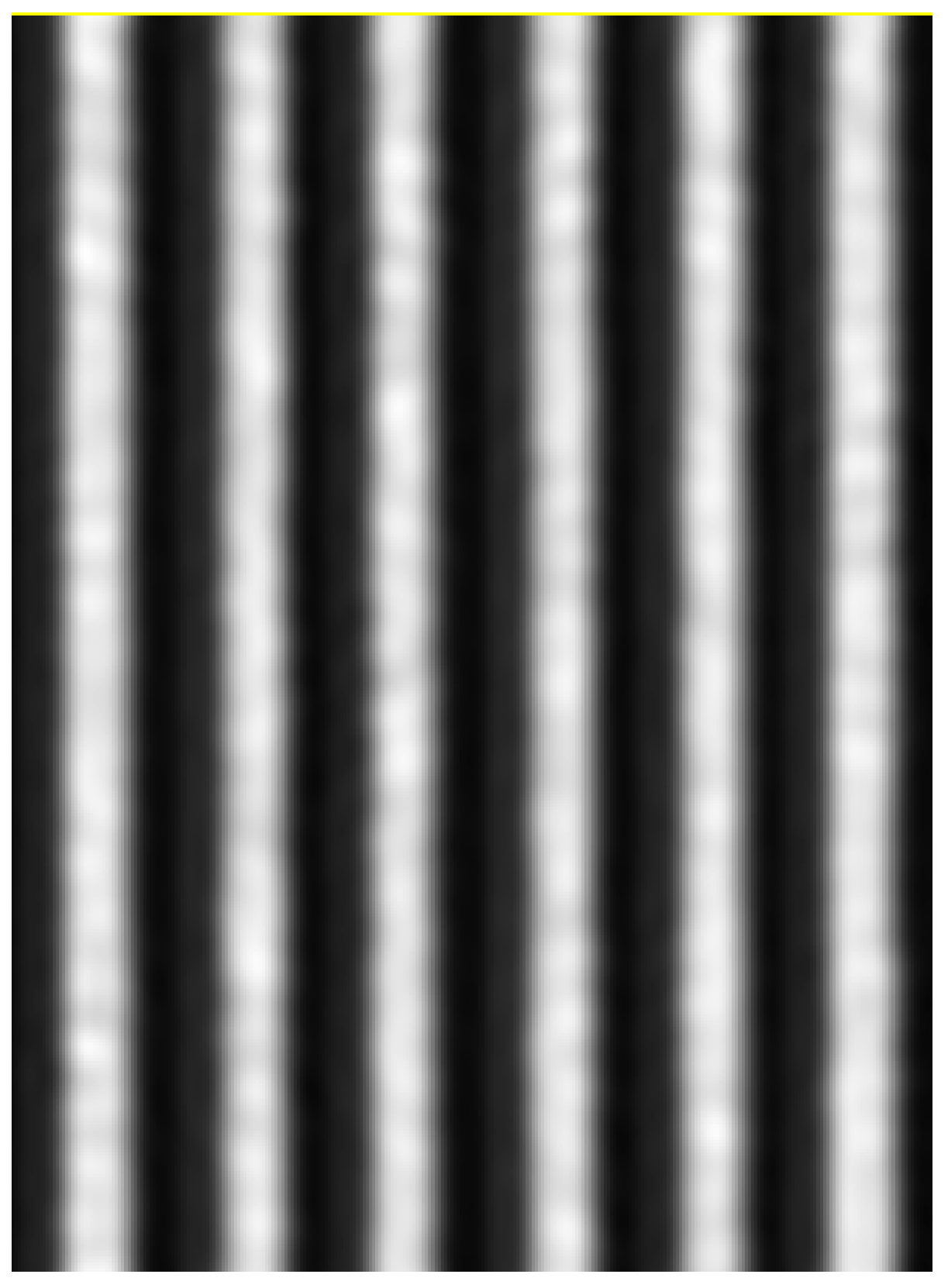


Naulleau and Gallatin, Fig. 3

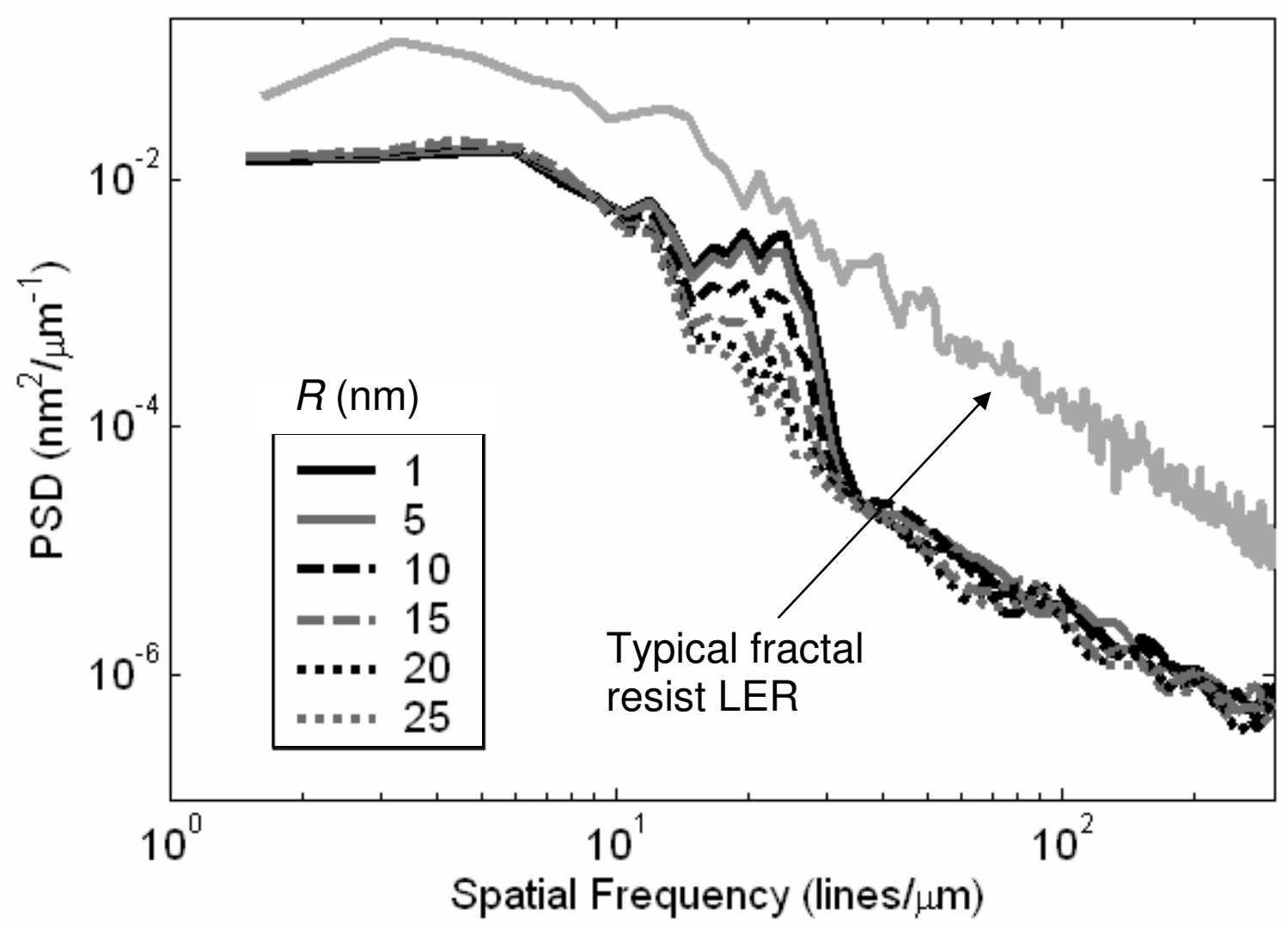


Naulleau and Gallatin, Fig. 4

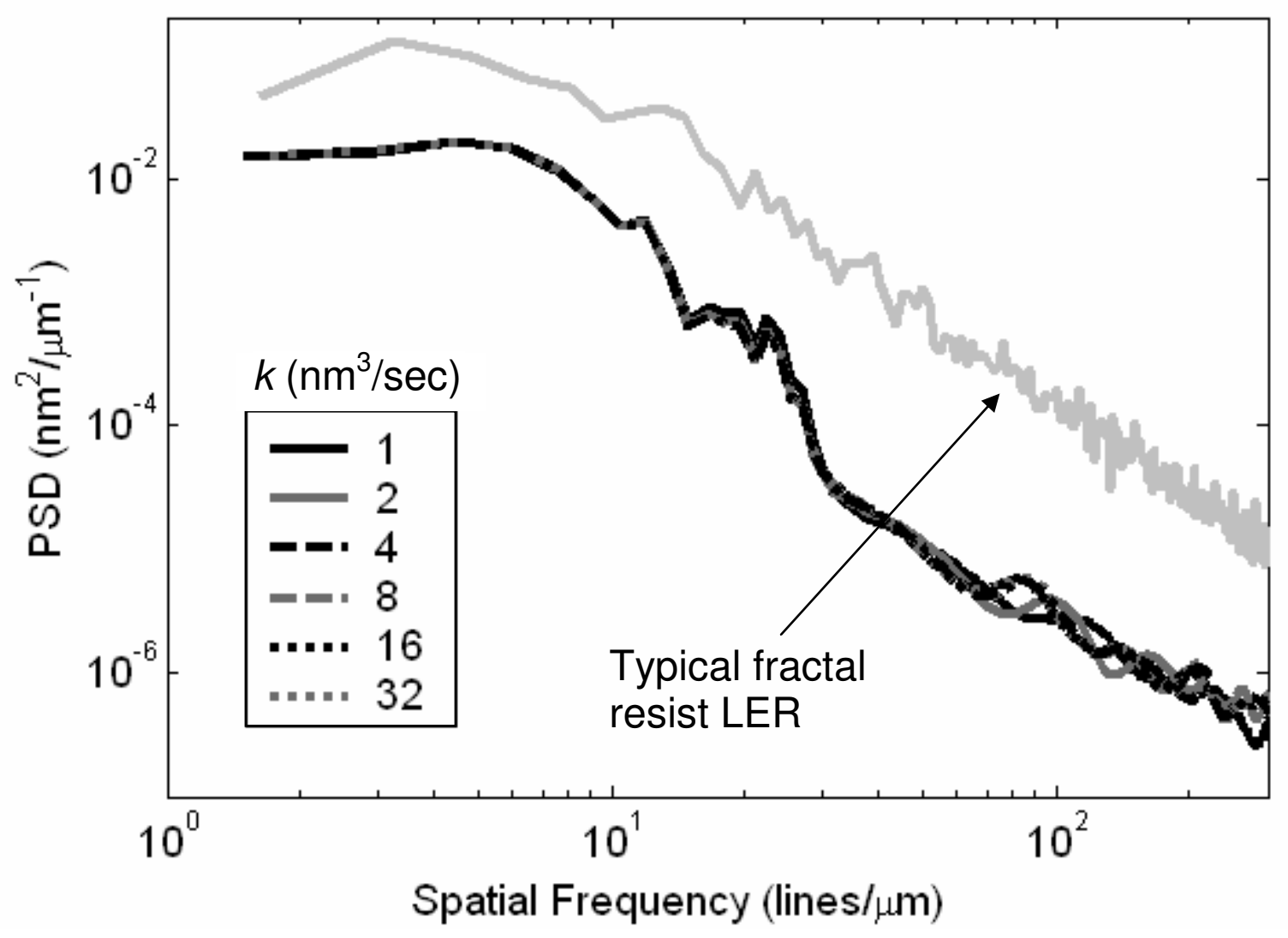



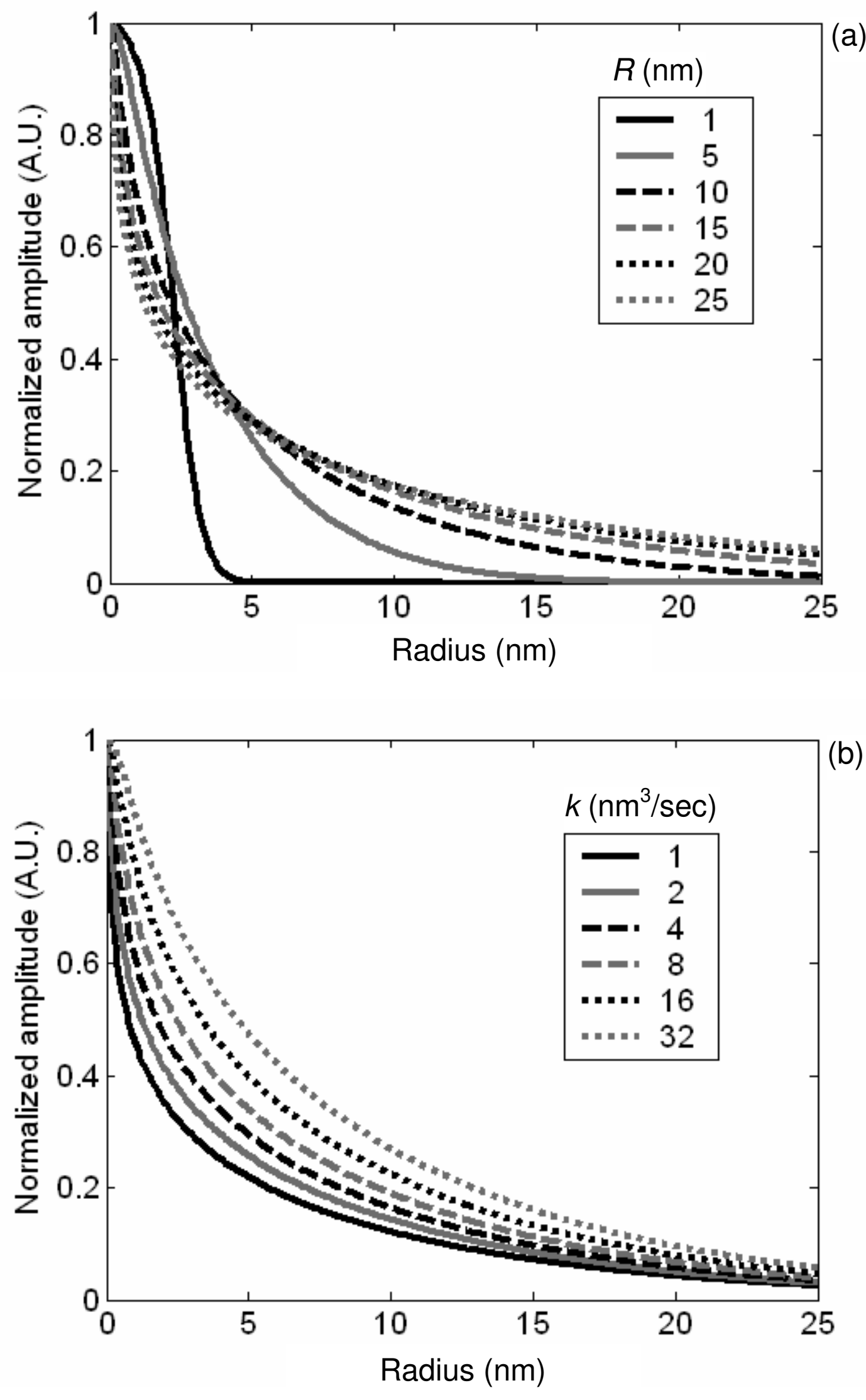
Naulleau and Gallatin, Fig. 6

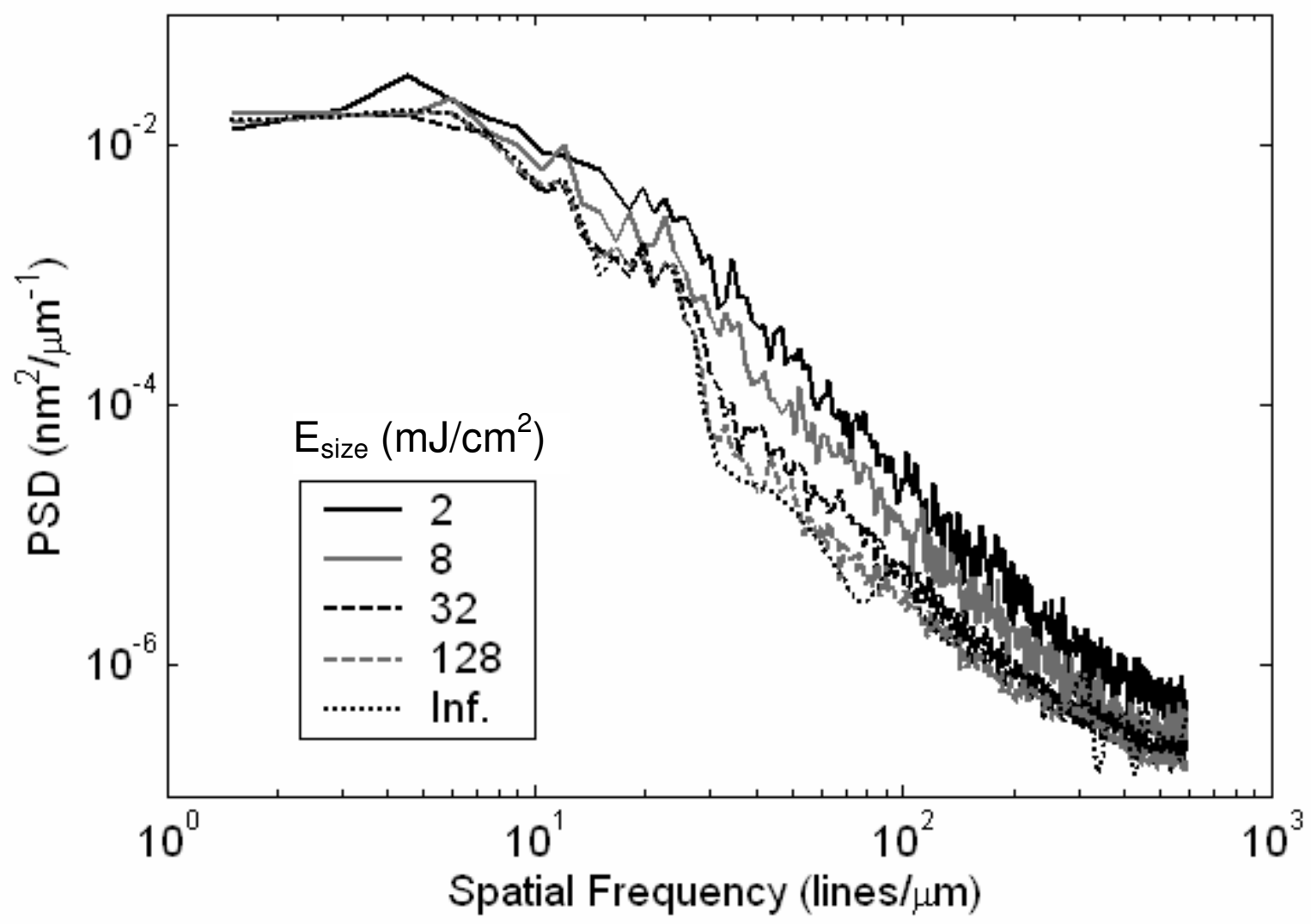


Naulleau and Gallatin, Table 1

\begin{tabular}{|c|c|c|c|c|c|}
\hline $\boldsymbol{k}\left(\mathbf{n m}^{2} / \mathbf{s e c}\right)$ & $\boldsymbol{R}(\mathbf{n m})$ & $\boldsymbol{\alpha}$ PSD & $\boldsymbol{\alpha}$ HHCF & LWR $(\mathbf{n m})$ & LER $(\mathbf{n m})$ \\
\hline NA & NA & 2.7 & 0.91 & $2.7 \pm 0.2$ & $1.8 \pm 0.1$ \\
\hline 4 & 1 & 3.3 & 0.84 & $2.7 \pm 0.2$ & $1.8 \pm 0.1$ \\
\hline 4 & 5 & 2.0 & 0.90 & $2.7 \pm 0.2$ & $1.8 \pm 0.1$ \\
\hline 4 & 10 & 2.4 & 0.86 & $2.7 \pm 0.2$ & $1.7 \pm 0.1$ \\
\hline 4 & 15 & 1.2 & 0.81 & $2.7 \pm 0.2$ & $1.7 \pm 0.1$ \\
\hline 4 & 20 & 1.5 & 0.78 & $2.8 \pm 0.2$ & $1.7 \pm 0.1$ \\
\hline 4 & 25 & 2.1 & 0.74 & $2.8 \pm 0.2$ & $1.6 \pm 0.1$ \\
\hline 1 & 15 & 1.1 & 0.80 & $2.7 \pm 0.2$ & $1.7 \pm 0.1$ \\
\hline 2 & 15 & 1.1 & 0.81 & $2.7 \pm 0.2$ & $1.7 \pm 0.1$ \\
\hline 4 & 15 & 1.1 & 0.80 & $2.7 \pm 0.2$ & $1.7 \pm 0.1$ \\
\hline 8 & 15 & 1.1 & 0.81 & $2.7 \pm 0.2$ & $1.7 \pm 0.1$ \\
\hline 16 & 15 & 1.1 & 0.81 & $2.7 \pm 0.2$ & $1.7 \pm 0.1$ \\
\hline 32 & 15 & 1.1 & 0.81 & $2.8 \pm 0.2$ & $1.7 \pm 0.1$ \\
\hline
\end{tabular}


Naulleau and Gallatin, Table 2

\begin{tabular}{|c|c|c|c|c|}
\hline $\begin{array}{c}\mathbf{E}_{\text {size }} \\
\left(\mathbf{m} \mathbf{J} / \mathbf{c m}^{2}\right)\end{array}$ & $\boldsymbol{\alpha}$ PSD & $\boldsymbol{\alpha}$ HHCF & LWR (nm) & LER (nm) \\
\hline 2 & 1.0 & 0.82 & $3.7 \pm 0.2$ & $2.4 \pm 0.1$ \\
\hline 4 & 1.0 & 0.81 & $3.1 \pm 0.2$ & $2.1 \pm 0.1$ \\
\hline 8 & 1.0 & 0.82 & $3.0 \pm 0.2$ & $1.9 \pm 0.1$ \\
\hline 16 & 1.2 & 0.83 & $2.7 \pm 0.2$ & $1.8 \pm 0.1$ \\
\hline 32 & 1.3 & 0.85 & $2.8 \pm 0.2$ & $1.8 \pm 0.1$ \\
\hline 64 & 1.5 & 0.88 & $2.6 \pm 0.2$ & $1.7 \pm 0.1$ \\
\hline 128 & 1.7 & 0.89 & $2.6 \pm 0.2$ & $1.7 \pm 0.1$ \\
\hline 256 & 1.8 & 0.91 & $2.6 \pm 0.2$ & $1.7 \pm 0.1$ \\
\hline$\infty$ & 2.4 & 0.86 & $2.7 \pm 0.2$ & $1.7 \pm 0.1$ \\
\hline
\end{tabular}

